\title{
The measurement of methacholine responsiveness in 5 year old children: three methods compared
}

\author{
N.M. Wilson, P. Bridge, S.B. Phagoo, M. Silverman
}

The measurement of methacholine responsiveness in 5 year old children: three methods compared. N.M. Wilson, P. Bridge, S.B. Phagoo, M. Silverman. CERS Journals Ltd 1995.

ABSTRACT: The aim of this study was to compare the feasibility of three techniques for measuring the response to bronchial challenge in young children: a direct airway measurement, the forced oscillation technique (FOT) for determining respiratory system resistance at 6 and $8 \mathrm{~Hz}$ (Rrs6 and Rrs8), and two indirect methods, the change in transcutaneous oxygen tension $\left(\mathrm{PtcO}_{2}\right)$ and the detection of wheeze on auscultation of the chest.

Thirty children aged $5 \mathrm{yrs}$, with a history of wheeze, and six asymptomatic controls, took part in a bronchial challenge test using methacholine administered by Wright nebulizer by the tidal-breathing method. The provocative concentration which produced a 35\% increase in Rrs6 (PC35Rrs6) and a 15\% decreases in $\mathrm{PtcO}_{2}$ $\left(\mathrm{PC} 15 \mathrm{PtcO}_{2}\right)$ were determined by interpolation, and the chest was auscultated after each dose of methacholine.

The FOT was found to be unreliable in this age group: in seven children, the data were technically unsatisfactory in the presence of induced bronchoconstriction, whilst in three children, changes in Rrs were inconsistent after challenge. The use of Rrs8 did not improve the detection of positive responses. $\mathrm{PC}_{15} \mathrm{Ptco}_{2}$ was measurable in 29 of 30 children, and in 18 of these PC35Rrs6 was also measurable. In no subject did a significant, sustained increase in Rrs occur during challenge in the absence of a significant change in $\mathrm{Ptco}_{2}$. Wheeze was audible in only 4 of $25(16 \%)$ of the positive and in no negative challenges.

With this protocol, we found the FOT to be unreliable and the auscultation method valueless and potentially dangerous, since marked falls in $\mathrm{PtcO}_{2}$ of up to $33 \%$ sometimes occurred in the absence of wheeze. The $\mathrm{Ptco}_{2}$ method seems to be the most technically reliable technique for measuring the response to bronchial challenge in 5 year old children. The underlying pathophysiology and diagnostic value of $\mathrm{PC}_{15} \mathrm{Ptco}_{2}$ values in young children remain to be established.

Eur Respir J., 1995, 8, 364-370.
Dept of Paediatrics and Neonatal Medicine, Royal Postgraduate Medical School, Hammersmith Hospital, London, UK.

Correspondence: N. Wilson

Dept of Paediatrics and Neonatal Medicine Royal Postgraduate Medical School Hammersmith Hospital

London W12 0NN

UK

Keywords: Bronchial responsiveness preschool children

Received: August 41994

Accepted after revision December 301994
Bronchial responsiveness has been extensively studied in school age children and in adults. Information is now becoming available about responsiveness in sedated infants, but there remains a gap in our understanding of its development between infancy and school-age, because of the difficulty in assessing the airway response in this age group. Recently, three methods of measuring the response to constrictor agents have been recommended for use in unsedated preschool children unable to cooperate with standard lung function testing. Firstly, the measurement of respiratory resistance using the forced oscillation technique (FOT) has been recommended for children as young as $2.5 \mathrm{yrs}$, because it only requires passive co-operation $[1,2]$. The second, an indirect method, makes use of the decrease in transcutaneous oxygen tension $\left(\mathrm{PtcO}_{2}\right)$ that occurs in association with induced bronchoconstriction [3-5]. This has been shown over a wide range of inhaled methacholine concentrations in asthmatic children and normal adults [4]. The third method relies on auscultation of the chest to detect the presence of rhonchi as an indication of induced airway narrowing $[6,7]$.

The three methods have been compared with standard lung function techniques in older children, and all have been found to give comparable results $[2,4,7]$, and to be repeatable $[1,5,7]$. All three methods have drawbacks: expensive equipment, indirect indicators of lung function, or a subjective measure. Before large population studies to determine the relationship of responsiveness to symptoms are carried out, it is necessary to have a technical appraisal of the lung function methods available in this age group. Therefore, in order to determine which of these three methods was the most technically reliable for use in young children, a comparison has been 
made between FOT to measure respiratory resistance at the two lowest practicable frequencies $(6$ and $8 \mathrm{~Hz})$ and two indirect methods, one employing changes in $\mathrm{PtcO}_{2}$ and the other auscultation of the chest during methacholine bronchial challenge in wheezy and healthy 5 year old children.

\section{Methods}

\section{Subjects}

Wheezing group. This group included 30 children (17 boys) with a history of wheeze, which had in the past been sufficiently troublesome to require admission to hospital, who were taking part in a longitudinal project of children admitted to hospital with wheeze in the first 2 yrs of life. At the time of study, eight had been symptom free for $>9$ months, 11 wheezed occasionally in association with viral infections, and 11 were considered to have asthma. All were studied within 2 months of their fifth birthday. Bronchodilators and cromoglycate were withheld for at least $12 \mathrm{~h}$ before each study, but topical corticosteroids (six children) were taken as usual.

Asymptomatic group. Six children with no history of atopic disease or lower respiratory symptoms were also enrolled. They were either children of staff members or unaffected siblings of children attending the asthma clinic. The mean (SD) age of these control children was $5.6(0.9)$ yrs.

Approval was obtained from the hospital Ethics Committee and parents gave their written consent.

\section{Challenge procedure}

A shortened validated protocol, suitable for use in preschool children was used [5]. Methacholine sulphate (0.5-32 g. $\left.l^{-1}\right)$ was inhaled using tidal-breathing for one minute via the same Wright nebulizer for each child (mean output (SD) $\left.0.14(0.02) \mathrm{ml} \cdot \mathrm{min}^{-1}\right)$. Quadrupling concentrations were administered at 5 min intervals, until the $\mathrm{PtcO}_{2}$ had fallen by $1 \mathrm{kPa}$ (about $10 \%$ ), after which increments were reduced to doubling concentrations. For safety reasons, the inhalations were stopped when the $\mathrm{PtcO}_{2}$ had fallen by at least $20 \%$, or the child was heard to wheeze or was dyspnoeic, or until the maximum concentration of methacholine had been given, whichever occurred the soonest.

\section{Assessment of the response}

The respiratory resistance was measured using a system designed and made by LANDSÉR et al. [8]. Pseudorandom noise oscillations $(4-48 \mathrm{~Hz})$ were generated by a loud speaker and superimposed on tidal-breathing at the mouth of the seated child. The nose was clipped and the cheeks and the floor of mouth were supported as the child breathed through a mouthpiece. Respiratory system resistance was determined at $6 \mathrm{~Hz}$ (Rrs6) and $8 \mathrm{~Hz}$ (Rrs8) during a $16 \mathrm{~s}$ sampling period from the regression of resistance against oscillation frequency. Data were accepted only if the coherence of the signal (a measure of interference with the signal from tidal-breathing and other sources of "noise") was $\geq 0.95$. Six baseline measurements were made, from which the mean value and coefficient of variation were calculated for each subject. Three minutes after each methacholine inhalation, the Rrs measurements were repeated until at least one coherent value was obtained and the first of these was used $[4,5]$.

The $\mathrm{PtcO}_{2}$ was measured with an electrode $\left(\mathrm{Cutan} \mathrm{PO}_{2}\right.$ Monitor 820, Kontron) attached to the anterior chest wall after calibration in air, operating at $44^{\circ} \mathrm{C}$. After a 20 min equilibration period, readings were taken manually on the minute. Eight baseline measurements were used to calculate the mean value and the coefficient of variation for each subject. After methacholine, readings were recorded every minute for $3 \mathrm{~min}$. The lowest value at the second or third minute was used $[4,5]$.

Following the protocol of NovisKi et al. [7] the chest was auscultated for $20 \mathrm{~s}$ using a regular paediatric stethoscope over the trachea and both lung fields posteriorly, before and $30 \mathrm{~s}, 1 \mathrm{~min}$ and $2.5 \mathrm{~min}$ after methacholine inhalation in 29 of the 36 challenges. The presence of audible wheeze (rhonchi) constituted a positive result. A note was made of any induced coughing and breathlessness or respiratory distress.

\section{Analysis of results}

Following methacholine inhalation, an increase in Rrs6 and Rrs8 of at least 35\% (double the upper 95\% confidence limit for the coefficient of variation (COV) for the group) was taken to indicate a positive response. For change in $\mathrm{PtcO}_{2}$, a threshold value of $15 \%$ was used, although this was 4.5 times the upper $95 \%$ confidence limit for the group $\mathrm{COV}$, as an approximately equivalent provocative concentration was required in order to make a comparison between the two methods. The concentrations of methacholine causing a $35 \%$ increase in Rrs6 (PC35Rrs6) and a 15\% decrease in $\mathrm{PtcO}_{2}\left(\mathrm{PC}_{15} \mathrm{PtcO}_{2}\right)$ were calculated from the log dose-response curve by linear interpolation. The two were compared by Student's paired t-test after logarithmic transformation. Values of $\mathrm{p}$ less than 0.05 were considered to be statistically significant.

\section{Results}

Wheezy group

Comparison between forced oscillation technique (Rrs) and $\mathrm{PtcO}_{2}$. For repeated baseline measurements, the mean 
Table 1. - Individual lung function data

\begin{tabular}{|c|c|c|c|c|c|c|c|c|c|c|}
\hline \multirow{2}{*}{$\begin{array}{l}\text { Subject } \\
\text { No. }\end{array}$} & \multirow[t]{2}{*}{ Sex } & \multicolumn{4}{|c|}{ Baseline } & \multirow[t]{2}{*}{$\begin{array}{l}\text { Maximum }[\mathrm{Mch}] \\
\text { inhaled } \mathrm{g} \cdot l^{-1}\end{array}$} & \multicolumn{3}{|c|}{$\begin{array}{c}\text { Change at max }[\mathrm{Mch}] \\
\%\end{array}$} & \multirow{2}{*}{$\begin{array}{c}\text { Wheeze } \\
+/ 0\end{array}$} \\
\hline & & $\begin{array}{c}\text { Rrs6 } \\
\% \text { pred }^{\$}\end{array}$ & $\mathrm{COV}$ & $\begin{array}{l}\mathrm{PtcO}_{2} \\
\mathrm{kPa}\end{array}$ & $\underset{\%}{\mathrm{COV}}$ & & Rrs6 & Rrs8 & $\mathrm{PtcO}_{2}$ & \\
\hline \multicolumn{11}{|c|}{ Study group (wheezy) } \\
\hline 1 & $\mathrm{~F}$ & 108 & 11 & 11.8 & 0.9 & 16 & $\mathrm{NC}$ & 17 & $-21 * \dagger$ & + \\
\hline 2 & $\mathrm{~F}$ & 93 & 8.0 & 9.6 & 0.8 & 4 & 8 & 13 & $-29 *$ & 0 \\
\hline 3 & M & 80 & 7.3 & 10.4 & 2.0 & 16 & $50 *$ & $36^{*}$ & $-20^{*}$ & + \\
\hline 4 & $\mathrm{~F}$ & 105 & 12 & 10.4 & 0.8 & 8 & $\mathrm{NC}$ & 2 & $-20 * \dagger$ & 0 \\
\hline 5 & $\mathrm{~F}$ & 101 & 11 & 11.1 & 1.7 & 8 & 15 & 25 & $-33^{*}$ & 0 \\
\hline 6 & M & 88 & 13 & 9.7 & 1.9 & 32 & $64 *$ & $47 *$ & $-22 *$ & 0 \\
\hline 7 & M & 102 & 7 & 10.7 & 1.5 & 16 & $46^{*}$ & 32 & $-25^{*}$ & 0 \\
\hline 8 & M & 69 & 11 & 10.5 & 0.8 & 32 & $92 *$ & $71^{*}$ & -6 & 0 \\
\hline 9 & $\mathrm{~F}$ & 99 & 11 & 8.4 & 2.8 & 16 & $70 *$ & $52 *$ & $-17 *$ & + \\
\hline 10 & $\mathrm{~F}$ & 159 & 10 & 9.9 & 0.8 & 16 & $17^{\dagger}$ & 18 & $-27 *$ & 0 \\
\hline 11 & $\mathrm{~F}$ & 82 & 14 & 11.5 & 0.4 & 32 & $\mathrm{NC}^{\dagger}$ & $56^{*}$ & $-19 * \dagger$ & 0 \\
\hline 12 & M & 120 & 21 & 8.8 & 0.7 & 32 & $\mathrm{NC}$ & NA & $-27 * \dagger$ & 0 \\
\hline 13 & M & 72 & 11 & 11.7 & 0.9 & 32 & $96^{*}$ & $88^{*}$ & $-18^{*}$ & 0 \\
\hline 14 & M & 93 & 17 & 10.0 & 1.0 & 32 & 32 & 31 & $-26^{*}$ & 0 \\
\hline 15 & M & 84 & 9 & 12.2 & 1.3 & 16 & $72 *$ & $61 *$ & $-20 *$ & 0 \\
\hline 16 & M & 107 & 13 & 11.5 & 0.8 & 32 & $62 *$ & 33 & $-23^{*}$ & NA \\
\hline 17 & M & 105 & 12 & 10.9 & 2.8 & 16 & $69^{*}$ & NA & $-39 *$ & NA \\
\hline 18 & M & 88 & 10 & 12.1 & 1.1 & 16 & $\mathrm{NC}$ & NA & $-29 *$ & NA \\
\hline 19 & M & 89 & 8 & 10.0 & 1.7 & 8 & $60 *$ & $54 *$ & $-22 *$ & NA \\
\hline 20 & M & 92 & 13 & 11.0 & 1.3 & 32 & $\mathrm{NC}$ & 26 & $-25^{* \dagger}$ & NA \\
\hline 21 & M & 101 & 9 & 12.0 & 1.0 & 32 & $80 *$ & $54 *$ & $-24^{*}$ & NA \\
\hline 22 & M & 88 & 4 & 8.3 & 1.5 & 32 & $43^{*}$ & $38 *$ & -13 & 0 \\
\hline 23 & $\mathrm{~F}$ & 98 & 10 & 8.6 & 0.6 & 32 & $53 *$ & $53 *$ & $-22 *$ & 0 \\
\hline 24 & $\mathrm{~F}$ & 104 & 7 & 12 & 4.0 & 16 & $56^{*}$ & $58 *$ & $-22 *$ & 0 \\
\hline 25 & $\mathrm{~F}$ & 129 & 9 & 10.5 & 3.3 & 16 & $37 *$ & 26 & $-29 *$ & 0 \\
\hline 26 & M & 83 & 10 & 10.5 & 3.3 & 16 & $\mathrm{NC}$ & $35^{*}$ & $-28 * \dagger$ & 0 \\
\hline 27 & $\mathrm{~F}$ & 139 & 10 & 10.2 & 1.4 & 32 & 20 & 24 & $-20^{*}$ & 0 \\
\hline 28 & $\mathrm{~F}$ & 97 & 12 & 10.7 & 1.2 & 1.0 & $72 *$ & $57 *$ & $-20 *$ & 0 \\
\hline 29 & M & 115 & 21 & 9.5 & 1.2 & 32 & 13 & 7 & -11 & NA \\
\hline 30 & M & 101 & 15 & 10.8 & 1.2 & 32 & 6 & NA & -9 & 0 \\
\hline Mean & & 99.7 & 11.2 & 10.6 & 1.5 & - & - & - & - & - \\
\hline $\mathrm{SD}$ & & 18.9 & 3.35 & 1.10 & 0.90 & - & - & - & - & - \\
\hline \multicolumn{11}{|c|}{ Control group } \\
\hline 31 & M & 115 & 21 & 9.5 & 1.2 & 32 & 0 & - & 11 & 0 \\
\hline 32 & $\mathrm{~F}$ & 81 & 10 & 11.1 & 4 & 32 & 14 & - & 11 & 0 \\
\hline 33 & M & 90 & 6 & 9.8 & 0.5 & 32 & 3 & - & 0 & 0 \\
\hline 34 & $\mathrm{~F}$ & 119 & 8 & 11.5 & 0.7 & 8 & $14 *$ & - & $20 *$ & 0 \\
\hline 35 & M & 85 & 11 & 11.6 & 0.8 & 32 & $105 *$ & - & $25^{*}$ & + \\
\hline 36 & M & 101 & 11 & 10.7 & 2 & 16 & $\mathrm{NC}$ & - & $27 *$ & 0 \\
\hline Mean & & 98.5 & 11.2 & 10.7 & 1.5 & - & - & - & - & - \\
\hline $\mathrm{SD}$ & & 15.8 & 5.2 & 0.9 & 1.0 & - & - & - & - & - \\
\hline \multicolumn{11}{|c|}{ Total group } \\
\hline Mean & & 99 & 11.2 & 10.5 & 1.5 & & & & & \\
\hline SD & & 18 & 3.9 & 1.1 & 1.0 & & & & & \\
\hline
\end{tabular}

COV: coefficient of variation; Mch: methacholine; NA: not available; NC: not coherent; Rrs6: respiratory system resistance at $6 \mathrm{~Hz}$; Rrs8: respiratory system resistance at $8 \mathrm{~Hz} ; \mathrm{PtcO}_{2}$ : transcutaneous oxygen tension; \$: taken from [9]. +: present; 0: absent; M: male; F: female. ': a significant increase in Rrs6 had occurred at a lower concentration of methacholine; *: significant change in $\operatorname{Rrs} 6(\geq 35 \%)$ and $\mathrm{PtcO}_{2}(\geq 15 \%)$.

(SD) intrasubject COV of Rrs6 was $11.2 \%$ (3.35) and of $\mathrm{PtcO}_{2}$ was $1.5 \%$ (0.9). Bronchial challenge was negative in two subjects (Nos. 29 and 30), as judged by all methods of assessment. A significant change in Rrs6 or
$\mathrm{PtcO}_{2}$ or both was seen in the other 28 subjects at or below the maximum concentration of methacholine inhaled (table 1). A significant change in both of these indices occurred in 14 subjects $(50 \%)$. In a further 12 
subjects, a fall in $\mathrm{PtcO}_{2}$ of at least $15 \%$ occurred in the absence of a significant change in Rrs6; in seven of them the Rrs6 result was repeatedly incoherent (low signal: noise ratio). In two subjects (subjects Nos. 10 and 11,

a) Subject No. 3

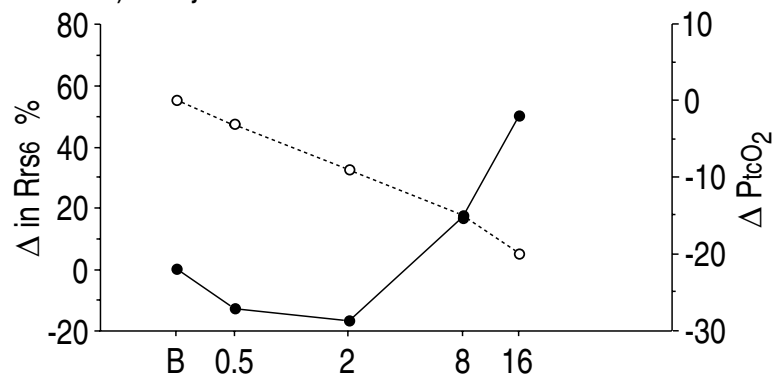

b) Subject No. 2

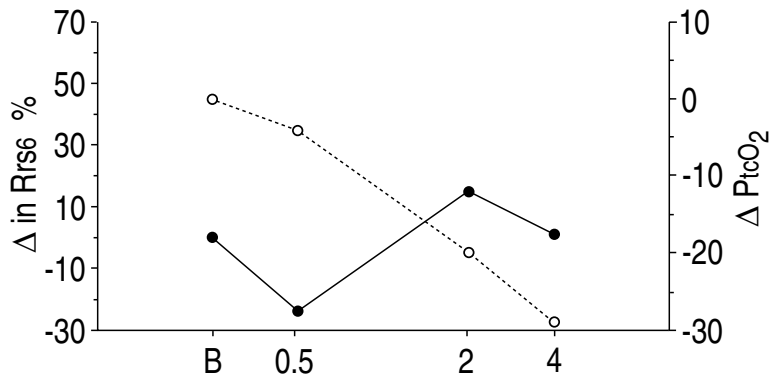

c) Subject No. 8

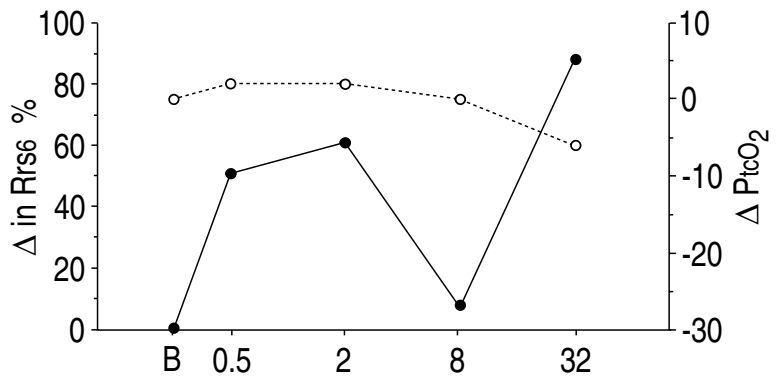

d) Subject No. 10

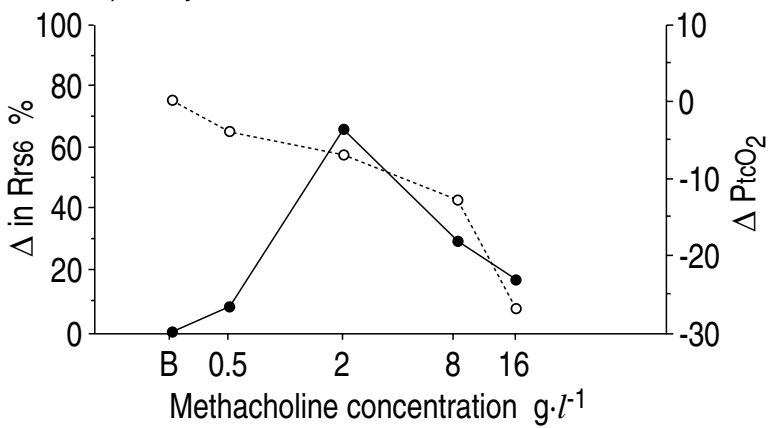

Fig. 1. - Varying responses in four subjects of Rrs6 (- - $)$ and $\mathrm{PtcO}_{2}$ (-O-) during methacholine challenge in four subjects. a) Subject No. 3 showing correspondence between Rrs6 and $\mathrm{PtcO}_{2}$. b) Subject No. 2 had a fall in $\mathrm{PtcO}_{2}$ without significant rise in Rrs6. c) Subject No. 8 showed a variable increase in Rrs6 but no change in $\mathrm{PtcO}_{2}$. d) Subject No. 10 had a significant change in $\mathrm{PtcO}_{2}$ and variable Rrs6 responses, resulting in discordant PC35Rrs6 and $\mathrm{PC} 15 \mathrm{PtcO}_{2}$. Rrs6: respiratory system resistance at $6 \mathrm{~Hz} ; \mathrm{PtcO}_{2}$ : transcutaneous oxygen tension; $\mathrm{B}$ : baseline; $\mathrm{PC} 35 \mathrm{Rrs6}$ : provocative concentration producing a 35\% increase in Rrs6; $\mathrm{PC} 15 \mathrm{PtcO}_{2}$ : provocative concentration producing a $15 \%$ decrease in $\mathrm{PtcO}_{2}$.

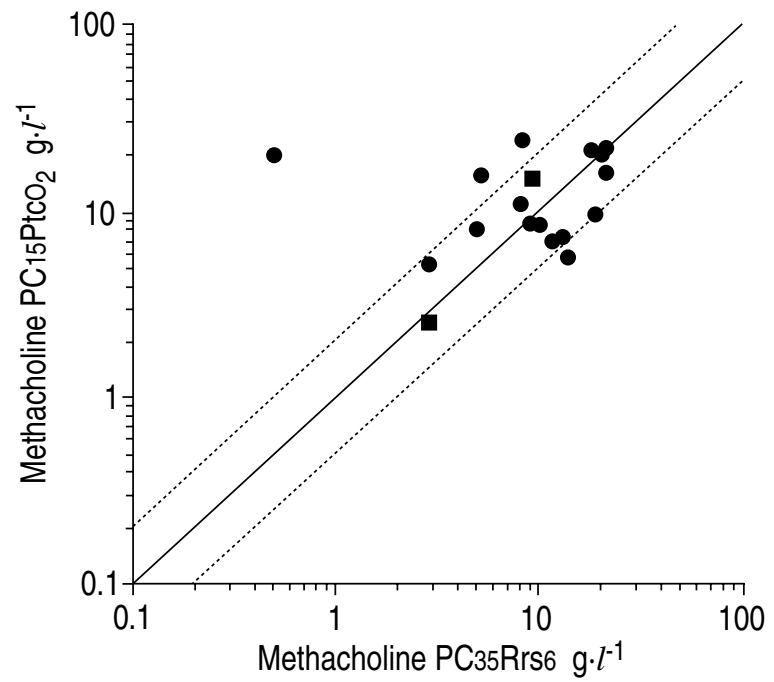

Fig. 2. - Comparison of PC35Rrs6 and $\mathrm{PC} 15 \mathrm{PtcO}_{2}$ in the 18 subjects in whom both were measurable, showing line of identify and range of one doubling dilution. $\bullet$ : study group; $\mathbf{\square}$ : control subjects. For abbreviations see legend to figure 1 .

fig. 1 and table 1), apparently significant increases in Rrs6 had been noted at low concentrations of methacholine, but at the final concentration the Rrs6 result was either incoherent or had reverted to a value which was not significantly higher than baseline.

In only two subjects were significant increases in Rrs6 not accompanied by a $15 \%$ fall in $\mathrm{PtcO}_{2}$. In one of these, the $\mathrm{PtcO}_{2}$ fell by $13 \%$ (more than six times the baseline COV), and in the other the changes in Rrs6 had shown a very variable course with increasing concentrations of methacholine (subject No. 8) (fig. 1). Thus, in no subject, did we find a significant and sustained increase in Rrs6 in the absence of a significant fall in $\mathrm{PtcO}_{2}$.

\section{$\operatorname{Rrs} 8$ versus $\operatorname{Rrs} 6$}

Rrs8 measurement (mean intrasubject COV 10.4\%) was available in 25 of 28 subjects with positive bronchial challenge. The use of Rrs8 instead of Rrs6 to assess the response at the maximal methacholine inhalation did not increase the overall positive response rate $(57: 58 \%)$ or the concordance with $\mathrm{PtcO}_{2}\left(\mathrm{PtcO}_{2}\right.$ and Rrs6 positive in $50 \%$ compared to $\mathrm{PtcO}_{2}$ and $\mathrm{Rrs} 8$ in $48 \%$ ). There were discrepancies in some individuals. In five subjects with incoherent Rrs6 results, coherent values for Rrs8 were obtained, but in another three, significant changes in Rrs6 were not correspondingly associated with significant changes in Rrs8. In the 20 of 28 subjects in whom both Rrs6 and Rrs8 data were available and coherent at the highest inhaled methacholine concentration, the mean (SD) change in Rrs6 of 51 (26)\% was significantly greater than the mean change in Rrs8 of $41(20) \%$ ( $\mathrm{p}<0.01$ by paired t-test), reflecting the negative frequency dependence of resistance during bronchoconstriction.

Auscultation. Wheezing was only noted during 3 of the 21 challenges deemed positive by $\mathrm{PtcO}_{2}$ or Rrs6 (table 1). 
Four children showed signs of some respiratory distress after their final methacholine dose, but in only one were rhonchi detected. Most children coughed 1-3 min after methacholine at doses which were associated with a fall in $\mathrm{PtcO}_{2}$.

\section{Asymptomatic group}

Comparison between forced oscillation technique (Rrs) and $\mathrm{PtcO}_{2}$. The control group did not differ from the wheezy group in respect of mean baseline Rrs6 or $\mathrm{PtcO}_{2}$ (table 1). During methacholine challenge, a significant fall in $\mathrm{PtcO}_{2}$ occurred in three subjects, and in two of them, this was associated with significant increases in Rrs6; in the third, the Rrs6 measurement was incoherent at the maximal concentration of methacholine. One child was heard to wheeze (table 1).

\section{PC35Rrs6 and $\mathrm{PC}_{15} \mathrm{Ptco}_{2}$ in both groups}

It was possible to calculate both the PC35Rrs6 and $\mathrm{PC} 15 \mathrm{PtcO}_{2}$ in 18 subjects (including subjects 34 and 35). There was no significant difference between the geometric means: PC35Rrs6 9.6 g. $l^{-1} ; \mathrm{PC}_{15} \mathrm{PtcO}_{2} 11.7 \mathrm{~g} \cdot l^{-1}$. The difference between the two was within one doubling dilution of methacholine in 14 of the 18 subjects, and within 1.5 doubling dilutions in a further three (fig. 2). In the remaining subject (subject No. 11, table 1) the difference was $>5$ doubling dilutions (fig. 2). When this outlier was excluded, there was a significant correlation between the two groups $\left(r^{2}, \operatorname{adj} .=0.3 ; p=0.02\right)$.

\section{Discussion}

In this age group, there is no well-tried method of assessing the response to bronchial challenge which can be considered as the "gold standard". The measurement of Rrs6 by the forced oscillation technique (FOT) might have been considered to represent the reference method, as it had been well-standardized in older subjects $[1,2,8]$, and is recommended for use as a method of measuring lung function in preschool children $[1,2]$. In the present study, however, it was not always reliable. Measurements were at times repeatedly incoherent (i.e. low signal:noise ratio) especially at the maximum methacholine concentration, when the $\mathrm{PtcO}_{2}$ had fallen. At other times no rise in resistance was detected, even when a child was clearly dyspnoeic and hypoxic. The $\mathrm{PtcO}_{2}$ reflecting ventilation perfusion mismatch [10] was easily the most sensitive method, although, because of problems with the FOT, the correspondence between changes in Rrs6 and $\mathrm{PtcO}_{2}$ was much less than that found in older children and adults [4].

Combining wheezy and asymptomatic groups, there were large falls in $\mathrm{PtcO}_{2}$ without detectable changes in Rrs6 in 13 subjects. In eight this was due to the inability to obtain a coherent value. In five cases, however, technically satisfactory results for Rrs6 were obtained but no increase was seen. The possibility arises, therefore, that the observed falls in $\mathrm{PtcO}_{2}$ could be independent of an effect of methacholine on the airways, for example, due to altered skin perfusion. In a previous comparison of Rrs6 and $\mathrm{PtcO}_{2}$ during methacholine challenge in older asthmatic children and normal adults, no fall in $\mathrm{PtcO}_{2}$ occurred in the absence of significantly increased Rrs6, over a very wide range of inhaled methacholine concentrations $\left(0.5-256 \mathrm{~g} \cdot \mathrm{l}^{-1}\right)$ [4]. By including a group of asymptomatic children, we had hoped to demonstrate no change in $\mathrm{PtcO}_{2}$ during negative studies. However, there was a high rate of response in these children, confirming the findings of other studies that in this age group methacholine responsiveness is neither specific for asthma nor related to the severity of symptoms $[11,12]$. We failed, however, to conclusively exclude a nonairway cause for the falls in $\mathrm{PtcO}_{2}$ in the five subjects without a significant rise in Rrs6 (table 1: subjects Nos. 2, 5, 14, 27 ). This is unlikely, as it would imply that these young children had a different systemic response to inhaled methacholine from the adults, who inhaled methacholine in very much higher concentrations [4].

There are several theoretical reasons for the less reliable performance of FOT in detecting acute bronchoconstriction in these young children. The measurement of Rrs includes both upper and lower airway resistance. The dissipation of the oscillatory signal in the upper airways, notably the cheeks and floor of the mouth, increases in the presence of a high level of lower airway resistance, as found in young children. This effect increases the upper airway contribution to the total resistance, so making it more difficult to detect induced changes in lower airway resistance during challenge, as well as exaggerating the negative frequency dependence of resistance [13]. The use of a lower oscillation frequency should increase the sensitivity of the FOT to detect bronchoconstriction in the presence of this negative frequency dependence, but the lower the frequency, the greater is the likelihood of interference with the oscillatory signal by breathing, leading to poor coherence. At a frequency of $6 \mathrm{~Hz}$, little interference was seen at rest in our study, but incoherent values of Rrs6 were a problem during bronchoconstriction in eight children. Increasing the frequency of the oscillatory signal to $8 \mathrm{~Hz}$ improved the number of studies with coherent values but reduced the sensitivity of the method to detect bronchoconstriction, because of the negative frequency dependence of resistance during induced bronchoconstriction. This difference between Rrs6 and Rrs8 in detecting bronchoconstriction has been reported previously [2].

Another problem with the use of oscillatory resistance is that it takes no account of changes in lung volume. Changes in functional residual capacity during challenge [14] could have caused underestimation of the change in Rrs. Using forced oscillations over a frequency spectrum of $4-52 \mathrm{~Hz}$ it is possible additionally to measure reactance, frequency dependence of resistance and resonant frequency [1]. None of these variables were sufficiently reproducible under baseline conditions to allow the calculation of a threshold change with which to describe the response to methacholine inhalation. This 
was disappointing, particularly as we had hoped to find a correlation between frequency dependence of resistance, as a reflection of ventilatory maldistribution, and falls in $\mathrm{PtcO}_{2}$.

In a previous comparison of Rrs6 and $\mathrm{PtcO}_{2}$ during methacholine challenge, we found that a $40 \%$ increase in Rrs6 was equivalent to a $20 \%$ fall in $\mathrm{PtcO}_{2}$ [4]. In the present study, only a minority of children achieved a $40 \%$ increase in resistance; therefore, to avoid multiple extrapolations, the PC35Rrs6 and $\mathrm{PC} 15 \mathrm{PtcO}_{2}$, which we knew from previous experience were equivalent, were compared. A 35\% increase in Rrs6 was twice the upper $95 \%$ confidence limit for COV for the group and at least twice the COV for Rrs6 in all but three of the subjects (Nos. 12, 19 and 31) (table 1). In fact, in none of the latter was a PC35Rrs6 obtained. In the 18 in whom both PC35Rrs6 and $\mathrm{PC}_{15} \mathrm{PtcO}_{2}$ were obtained, there was a reasonable correlation between the two in all but one subject (fig. 2). Inspection of the dose-response curve of this subject suggested that the Rrs6 measurement was unreliable, in that following the early significant rise in Rrs6 five further doubling increases in methacholine concentration did not produce any evidence of respiratory distress, and the accompanying changes in Rrs6 were variable. Thus, apart from one child with unreliable Rrs6 measurements, PC35Rrs6 was approximately equivalent to $\mathrm{PC} 15 \mathrm{PtcO}_{2}$, with no significant difference in mean values.

Induced bronchoconstriction was associated with coughing. It is possible that deep breathing during a bout of coughing could affect the degree of ventilation/perfusion V/Q mismatch associated with bronchoconstriction. Repeated measurements of $\mathrm{PtcO}_{2}$ were very reproducible, so a lower level of change, e.g. $10 \%$, could have been used to define a significant response. At this level of response, however, it would not have been possible to detect any change in Rrs6 for comparison.

Auscultation proved a very insensitive indicator of induced bronchoconstriction in this study. A fall in $\mathrm{PtcO}_{2}$ of up to $33 \%$ and a doubling in Rrs6 occurred in some subjects without audible wheeze. Auscultation was therefore neither a reliable nor a safe method of assessing bronchoconstriction, in marked contrast to the findings of Avital and coworkers [6], who detected wheeze in 12 of 15 children with an induced fall in forced expiratory volume in one second $\left(\mathrm{FEV}_{1}\right)$ of $>20 \%$. One possibility is that they induced a greater degree of bronchoconstriction, as others have suggested that at least a $27-30 \%$ fall in $\mathrm{FEV}_{1}$ is necessary before wheezing is heard $[15,16]$. Another possibility is that the higher output nebulizer used by Avital and coworkers, might result in a different site of aerosol deposition, which could explain the discrepancy in the sensitivity of auscultation in the two studies (14:80\%, respectively).

Like previous workers, we have shown a fall in $\mathrm{PtcO}_{2}$ to be a sensitive indicator of induced bronchoconstriction. Because of the variability of repeated measurements of Rrs, a threshold value of $40-50 \%$ increase in Rrs6 would be desirable, but at higher levels of Rrs6 the readings tended to underestimate the level of bronchoconstriction in these young children; hypoxia often occurred without such a target threshold being achieved. An additional problem was the interference with the signal by the high respiratory rate. Thus, in contrast to studies on older subjects, the measurement of Rrs6 was found to be unreliable in the presence of induced bronchoconstriction in this age group. Using this protocol, the detection of wheeze by auscultation was also an unsatisfactory and unsafe indicator of bronchoconstriction. Until a further technique is developed for use in very young children, the indirect measure of $\mathrm{PtcO}_{2}$ seems to be the method of choice for assessing the response to bronchial challenge in 5 year olds. However, direct comparison of results from children of different ages remains invalid, because of the effect of subject size on aerosol deposition and lung dose. Population studies are needed to determine whether any particular change in $\mathrm{PtcO}_{2}$ during challenge can be used to identify abnormality.

\footnotetext{
Acknowledgement: The authors are grateful to the National Asthma Campaign for supporting this research.
}

\section{References}

1. Duiverman EJ, Neijens HJ, van Stick R, van der SneeVan Smaalen M, Kerrebijn KF. Bronchial responsiveness in asthmatic children aged 3 to 8 years measured by forced pseudorandom noise oscillometry. Bull Eur Physiol Respir 1986; 22: 27-34.

2. Buhr W, Jorres R, Berdel D, Landser FJ. Correspondence between forced oscillation and body plethysmography during bronchoprovocation with carbachol in children. Pediatr Pulmonol 1990; 8: 280-288.

3. Mochizuki H, Mitshuhashi M, Tokuyama K, et al. A new method of estimating bronchial hyperresponsiveness in younger children. Ann Allergy 1985; 55: 162166.

4. Wilson NM, Phagoo SB, Silverman M. Use of transcutaneous oxygen tension, arterial oxygen saturation and respiratory resistance to assess the response to inhaled methacholine in asthmatic children and normal adults. Thorax 1991; 46: 430-437.

5. Phagoo SB, Wilson NM, Silverman M. Repeatability of methacholine challenge in asthmatic children measured by change in transcutaneous oxygen tension. Thorax 1992; 47: 804-808.

6. Avital A, Bar-Yishay E, Springer C, Godfrey S. Bronchial provocation tests in young children using tracheal auscultation. J Pediatr 1988; 112: 591-594.

7. Noviski N, Cohen L, Springer C, Bar-Yishay E, Avital $\mathrm{S}$, Godfrey S. Bronchial provocation determined by breath sounds compared with lung function. Arch Dis Child 1991; 66: 956-960.

8. Landsér FJ, Nagels J, Demedts M, Billiet L, Van de Woestijne KP. A new method to determine frequency characteristics of the respiratory system. J Appl Physiol 1976; 4: 101-106.

9. Duiverman EJ, Clement J, Van de Woestigne KP, Neigens HJ, Van de Bergh ACM, Kerrebijn KF. Forced oscillation technique: reference values for resistance and reactance over a frequency spectrum $2-68 \mathrm{~Hz}$ in healthy 
children 2.3-12.5 yrs. Bull Eur Physiopathol Respir 1985; 21: 171-178.

10. Wagner PD, Hedenstierna G, Bylin G. Ventilationperfusion inequality in chronic asthma. Am Rev Respir Dis 1987; 136: 605-612.

11. Wilson NM, Phagoo SB, Silverman M. Atopy, bronchial responsiveness and symptoms in wheezing 3 year olds. Arch Dis Child 1992; 67: 491-495.

12. Adachi Y, Murakami G, Matsuro M, et al. Longitudinal study of bronchial hyperreactivity in preschool children with bronchial asthma. Ann Allergy 1992; 68: 261266.

13. Pride NB. Forced oscillation techniques for measuring mechanical properties of the respiratory system. Thorax 1992; 47: 317-320.

14. Ding DJ, Martin JG, Macklem PT. The effects of lung volume on maximal methacholine-induced bronchoconstriction. J Appl Physiol 1987; 62: 1324-1330.

15. Spence DPS, Bentley S, Evans DH, Morgan MDL. Effect of methacholine-induced bronchoconstriction on the spectral characteristics of breath sounds in asthma. Thorax 1992; 47: 680-683.

16. Avital A, Noviski N, Bar-Yishay E, Springer E, Levy M, Godfrey S. Nonspecific bronchial reactivity in asthmatic children depends on severity but not on age. Am Rev Respir Dis 1991; 144: 36-38. 